\title{
Stability and Hopf Bifurcation Analysis of a Fractional-Order Epidemic Model with Time Delay
}

\author{
Zhen Wang ${ }^{1,2}$ and Xinhe Wang ${ }^{1}$ \\ ${ }^{1}$ College of Mathematics and Systems Science, Shandong University of Science and Technology, Qingdao 266590, China \\ ${ }^{2}$ College of Electrical Engineering and Automation, Shandong University of Science and Technology, Qingdao 266590, China \\ Correspondence should be addressed to Zhen Wang; wangzhen_sd@126.com
}

Received 13 January 2018; Accepted 14 June 2018; Published 3 July 2018

Academic Editor: Gisele Mophou

Copyright (c) 2018 Zhen Wang and Xinhe Wang. This is an open access article distributed under the Creative Commons Attribution License, which permits unrestricted use, distribution, and reproduction in any medium, provided the original work is properly cited.

\begin{abstract}
A fractional-order epidemic model with time delay is considered. Firstly, stability of the disease-free equilibrium point and endemic equilibrium point is studied. Then, by choosing the time delay as a bifurcation parameter, the existence of Hopf bifurcation is studied. Finally, numerical simulations are given to illustrate the effectiveness and feasibility of theoretical results.
\end{abstract}

\section{Introduction}

Mathematical model plays an important role in describing the dynamics of biological system [1-3]. The dynamics of the epidemic models have received much attention during the recent years, and to explain the disease spreading and control strategies a series of epidemic models [4-7] was proposed. A stochastic SIRS epidemic model was formulated in [8]; it investigated the effect of stochastic environmental variability on interpandemic transmission dynamics of influenza A. In [9], an age-structured SEIR epidemic model was considered. The authors investigated an SEIR model with varying population size and vaccination strategy in [10], and different threshold parameters were obtained to govern the disease eradication. Many models in biological mathematics involve some time delays. In biological dynamics, time delay was widely applied to reflect some biological facts, such as immunity period [11] and latent period of the disease [12]. An epidemic model with time delay was proposed in [13], and the model is shown as follows:

$$
\begin{aligned}
\frac{d S(t)}{d t}= & r S(t)\left(1-\frac{S(t)}{k}\right)-\beta S(t-\tau) I(t-\tau) \\
& +\mu S(t), \\
\frac{d I(t)}{d t}= & \beta S(t) I(t)-d I(t),
\end{aligned}
$$

where $S(t), I(t)$ represent the number of susceptible and infected population. $r$ represents the intrinsic birth rate constant, $k$ represents carrying capacity of susceptible population, $\beta$ represents the force of infection or the rate of transmission, $\mu$ represents immigration coefficient, $d$ represents death coefficient of $I(t)$, and $\tau$ is the latent period of the disease.

Fractional calculus is a generalization of classical differentiation and integration to arbitrary (noninteger) order [14]. In the past decades, fractional-order calculus garnered considerable attention and it was applied to various fields [15-21]. Recently, many investigators started to study the fractionalorder biological models [22-24]. The main reason is that fractional-order models are naturally related to systems with memory which exists in most biological systems $[25,26]$. In [27], the authors introduced a fractional-order prey-predator model and deal with the biological behaviors of the model. A fractional-order SIS model with variable population size is considered in [28], and the stability of equilibrium points is studied. A fractional-order model of two-species facultative mutualism with harvesting was presented in [29], and stability of the model was analyzed. In [30], the authors introduced a fractional-order epidemic model with vaccination; it shows that the stability region of the model is related to threshold-value $R_{0}$ and value of the fractional-order $\alpha$. A delayed fractional-order differential model of HIV infection of $\mathrm{CD} 4^{+}$ 
was investigated in [31]. In [32], a fractional-order prey-predator model with time delay and Monod-Haldane function was studied.

In this paper, a fractional-order epidemic model with time delay is studied. We investigate stability and bifurcation of the model with respect to basic reproduction number $R_{0}$, fractional-order $\alpha$ and time delay $\tau$. We provide theoretical analysis, using the eigenvalues method and linearization techniques and bifurcation method. The model is depicted as follows:

$$
\begin{aligned}
D^{\alpha} S(t)= & r S(t)\left(1-\frac{S(t)}{k}\right)-\beta S(t-\tau) I(t-\tau) \\
& +\mu S(t), \\
D^{\alpha} I(t)= & \beta S(t) I(t)-d I(t),
\end{aligned}
$$

where $\alpha \in(0,1], \tau \geq 0$, and $\alpha$ is in the sense of Caputo fractional derivatives. $S(t)=\phi_{1}(t), I(t)=\phi_{2}(t)$, and $t \in$ $[-\tau, 0]$.

The corresponding linearized system of (2) at any equilibrium point $\left(S^{*}, I^{*}\right)$ is defined as

$$
\begin{aligned}
D^{\alpha} S(t)= & \left(r+\mu-\frac{2 r S^{*}}{k}\right) S(t)-\beta I^{*} S(t-\tau) \\
& -\beta S^{*} I(t-\tau) \\
D^{\alpha} S(t)= & \beta I^{*} S(t)+\left(\beta S^{*}-d\right) I(t) .
\end{aligned}
$$

Taking Laplace transform [33] on both sides of (3), one obtains the characteristic matrix as follows:

$$
\Delta(s)=\left(\begin{array}{cc}
s-r-\mu-\frac{2 r S^{*}}{k}+\beta I^{*} e^{-s \tau} & \beta e^{-s \tau} S^{*} \\
-\beta I^{*} & s-\beta S^{*}+d
\end{array}\right) .
$$

The properties of eigenvalues of characteristic equation $\operatorname{det}(\Delta(s))$ indicate the stability of system (2).

The rest of the paper is organized as follows. In Section 2, some necessary definitions and notions are presented. In Section 3, stability and Hopf bifurcation of the equilibrium point are analyzed. Numerical simulations are given in Section 4 and some conclusions are given in Section 5.

\section{Preliminary}

There are three main definitions of fractional-order differential, that is, Riemann-Liouville, Grünwald-Letnikov, and Caputo's definitions. This paper is based on Caputo's definition.

Definition 1 (see [34]). The Caputo fractional derivative with order $\alpha$ of a continuous function $f: R^{+} \longrightarrow R$ is defined as follows:

$$
\begin{aligned}
& D_{t}^{\alpha} f(t) \\
& = \begin{cases}\frac{1}{\Gamma(n-\alpha)} \int_{0}^{t}(t-\xi)^{n-\alpha-1} f^{(n)}(\xi) d \xi, & n-1<\alpha<n, \\
\frac{d^{n}}{d t^{n}} f(t), & \alpha=n,\end{cases}
\end{aligned}
$$

where $\Gamma(z)=\int_{0}^{\infty} e^{-t} t^{z-1} d t$
Lemma 2 (see [35]). Considering the fractional differential system with the Caputo derivative,

$$
D^{\alpha} X=A X
$$

where $\alpha \in(0,1), X \in R^{n}$, and $A \in R^{n \times n}$. The characteristic equation of system (6) is $\operatorname{det}\left|s^{\alpha} I-A\right|=0$. If the real parts of all the eigenvalues of $A$ are negative, then the zero solution to system (6) is locally asymptotically stable.

Lemma 3 (see [33]). Considering the fractional delayed differential system with the Caputo derivative,

$$
\begin{aligned}
& D^{\alpha} X(t)=A X(t)+B X(t-\tau), \\
& \quad X(t)=\Phi(t), t \in[-\tau, 0],
\end{aligned}
$$

where $\alpha \in(0,1], X \in R^{n}, A, B \in R^{n \times n}$, and $\Phi(t) \in R_{+}^{n \times n}$. The characteristic equation of the system (7) is $\operatorname{det} \mid s^{\alpha} I-A-$ $B e^{-s \tau} \mid=0$. If all the roots of the characteristic equation have negative real parts, then the zero solution of system (7) is locally asymptotically stable.

\section{Main Results}

3.1. Basic Production Number and the Existence of the Equilibrium Point. Following from [16], system (2) has a disease-free equilibrium point $E_{0}=\left(S^{0}, I^{0}\right)=(k(r+\mu) / r, 0)$ and the basic reproduction number for the model is $R_{0}=k \beta(r+\mu) / r d$. Endemic equilibrium point is $E_{1}=\left(S^{1}, I^{1}\right)=(d / \beta,(k \beta(r+$ $\left.\mu)-r d) / k \beta^{2}\right)$. Obviously, $I^{1}=(k \beta(r+\mu)-r d) / k \beta^{2}=$ $\left(R_{0}-1\right) r d / k \beta^{2}$. Then we know that model (2) has an endemic equilibrium point $E_{1}\left(d / \beta,(k \beta(r+\mu)-r d) / k \beta^{2}\right)$ when $R_{0}>1$.

\subsection{Stability of the Disease-Free Equilibrium Point}

Theorem 4. The disease-free equilibrium point $E_{0}$ of system (2) is locally asymptotically stable if $R_{0}<1$.

Proof. The characteristic matrix of system (3) evaluated at the equilibrium point $E_{0}$ is

$$
\Delta(s)=\left[\begin{array}{cc}
s+r+\mu & \beta e^{-s \tau} \frac{k}{r}(r+\mu) \\
0 & s-\beta \frac{k}{r}(r+\mu)+d
\end{array}\right],
$$

and the characteristic equation is

$$
\left(s^{\alpha}+r+\mu\right)\left(s^{\alpha}-\beta \frac{k}{r}(r+\mu)+d\right)=0 .
$$

Let $s^{\alpha}=\lambda$; we can rewrite (9) as

$$
(\lambda+r+\mu)\left(\lambda-\beta \frac{k}{r}(r+\mu)+d\right)=0 .
$$

Clearly, $\lambda_{1}=-r-\mu<0, \lambda_{2}=(k \beta(r+\mu)-r d) / r$. When $R_{0}=k \beta(r+\mu) / r d<1$, we get $\lambda_{2}<0$. According to Lemma 2, the disease-free equilibrium point $E_{0}$ is locally asymptotically stable. This completes the proof. 
3.3. Stability of the Endemic Equilibrium Point. The characteristic matrix of system (3) evaluated at the equilibrium point $E_{1}$ is

$\Delta(s)$

$$
=\left(\begin{array}{cc}
s-\frac{k \beta(r+\mu)-2 r d}{k \beta}+e^{-s \tau} \frac{k \beta(r+\mu)-r d}{k \beta} & e^{-s \tau} d \\
-\frac{k \beta(r+\mu)-r d}{k \beta} & s
\end{array}\right),
$$

from which we have the characteristic equation

$$
s^{2 \alpha}+a_{1} s^{\alpha}+a_{2}=0
$$

where

$$
\begin{aligned}
& a_{1}=-\frac{k \beta(r+\mu)-2 r d}{k \beta}+e^{-s \tau} \frac{k \beta(r+\mu)-r d}{k \beta}, \\
& a_{2}=e^{-s \tau} d \frac{k \beta(r+\mu)-r d}{k \beta} .
\end{aligned}
$$

Theorem 5. When $\tau=0$, the endemic equilibrium point $E_{1}$ of system (2) is locally asymptotically stable if $R_{0}>1$.

Proof. Let $s^{\alpha}=\lambda$; we can rewrite (12) as

$$
\lambda^{2}+\frac{r d}{k \beta} \lambda+d \frac{k \beta(r+\mu)-r d}{k \beta}=0 .
$$

If $R_{0}>1$, one obtains $d((k \beta(r+\mu)-r d) / k \beta)=d\left(\left(R_{0}-\right.\right.$ 1) $r d / k \beta)>0$. Obviously, the two roots of (14) are negative. According to Lemma 2, the endemic equilibrium point $E^{*}$ is locally asymptotically stable. This completes the proof.

When $\tau>0$, (12) can be rewritten as

$$
s^{2 \alpha}+b_{1} s^{\alpha}+b_{2} e^{-s \tau} s^{\alpha}+b_{3} e^{-s \tau}=0,
$$

where

$$
\begin{aligned}
& b_{1}=-\frac{k \beta(r+\mu)-2 r d}{k \beta}, \\
& b_{2}=\frac{k \beta(r+\mu)-r d}{k \beta}, \\
& b_{3}=d \frac{k \beta(r+\mu)-r d}{k \beta} .
\end{aligned}
$$

Assume that (15) has a pair of pure imaginary roots $s_{1,2}= \pm i \omega$, $\omega>0$ and then substitute $s_{1}=i \omega$ into equation (15); one obtains

$$
(i \omega)^{2 \alpha}+b_{1}(i \omega)^{\alpha}+b_{2} e^{-i \omega \tau}(i \omega)^{\alpha}+b_{3} e^{-i \omega \tau}=0,
$$

and then separating the real and imaginary parts of (17) one has

$$
\begin{aligned}
& \omega^{2 \alpha} \cos \alpha \pi+b_{1} \omega^{\alpha} \cos \frac{\alpha \pi}{2} \\
& =-b_{2} \omega^{\alpha} \cos \left(\frac{\alpha \pi}{2}-\omega \tau\right)-b_{3} \cos \omega \tau, \\
& \omega^{2 \alpha} \sin \alpha \pi+b_{1} \omega^{\alpha} \sin \frac{\alpha \pi}{2} \\
& =-b_{2} \omega^{\alpha} \sin \left(\frac{\alpha \pi}{2}-\omega \tau\right)+b_{3} \sin \omega \tau .
\end{aligned}
$$

Squaring and adding the two equations in (18), we obtain

$$
\begin{gathered}
\omega^{4 \alpha}+2 b_{1} \cos \frac{\alpha \pi}{2} \omega^{3 \alpha}+\left(b_{1}^{2}-b_{2}^{2}\right) \omega^{2 \alpha} \\
-2 b_{2} b_{3} \cos \frac{\alpha \pi}{2} \omega^{\alpha}-b_{3}{ }^{2}=0 .
\end{gathered}
$$

Denote $h(\omega)=\omega^{4 \alpha}+2 b_{1} \cos (\alpha \pi / 2) \omega^{3 \alpha}+\left(b_{1}{ }^{2}-b_{2}{ }^{2}\right) \omega^{2 \alpha}-$ $2 b_{2} b_{3} \cos (\alpha \pi / 2) \omega^{\alpha}-b_{3}{ }^{2}$, where $-b_{3}{ }^{2}<0$; therefore (19) has one positive root at least. If $\omega_{1}, \omega_{2}, \omega_{3}, \omega_{4}$ are the roots of $h(\omega)$, we assume $\omega_{k}$ is positive. Substituting $\omega_{k}$ into (18), one obtains

$$
\begin{gathered}
\omega_{k}^{2 \alpha} \cos \alpha \pi+b_{2} \omega_{k}^{\alpha} \cos \left(\frac{\alpha \pi}{2}-\omega \tau\right) \\
=-b_{1} \omega_{k}^{\alpha} \cos \frac{\alpha \pi}{2}-b_{3} \cos \omega \tau, \\
\omega_{k}^{2 \alpha} \sin \alpha \pi+b_{2} \omega_{k}^{\alpha} \sin \left(\frac{\alpha \pi}{2}-\omega \tau\right) \\
=-b_{1} \omega_{k}^{\alpha} \sin \frac{\alpha \pi}{2}+b_{3} \sin \omega \tau .
\end{gathered}
$$

Squaring and adding the two equations in (20), one obtains

$$
\begin{aligned}
\omega_{k}^{4 \alpha}+ & 2 b_{2} \cos \left(\frac{\alpha \pi}{2}+\omega_{k} \tau\right) \omega_{k}^{3 \alpha}+\left(b_{2}^{2}-b_{1}^{2}\right) \omega_{k}^{2 \alpha} \\
& -2 b_{2} b_{3} \omega_{k}^{\alpha} \cos \left(\frac{\alpha \pi}{2}+\omega_{k} \tau\right)-b_{3}^{2}=0
\end{aligned}
$$

From $(21), \tau_{k}$ can be obtained

$$
\begin{aligned}
\tau_{k}^{j} & =\frac{1}{\omega_{k}}\left[\cos ^{-1}\left(\frac{\omega_{k}^{4 \alpha}+\left(b_{2}^{2}-b_{1}^{2}\right) \omega_{k}^{2 \alpha}-b_{3}^{2}}{2 b_{2} b_{3} \omega_{k}^{\alpha}-2 b_{2} \omega_{k}^{3 \alpha}}\right)-\frac{\alpha \pi}{2}\right. \\
& +2 j \pi], \quad j=0,1,2, \ldots
\end{aligned}
$$

Theorem 6. When $R_{0}>1$ and $h^{\prime}(\omega)>0$, the endemic equilibrium point $E_{1}$ of system (2) is locally asymptotically stable if $\tau<\tau_{0}$ and unstable if $\tau>\tau_{0}$, where $\tau_{0}=\min \left\{\tau_{k}^{j}\right\}$.

Proof. Let $F(s)=s^{2 \alpha}+b_{1} s^{\alpha}$ and $G(s)=b_{2} s^{\alpha}+b_{3}$; (12) reduces to

$$
F(s)+G(s) e^{-s \tau}=0 .
$$

Denote $h(z)=z^{4 \alpha}+2 b_{1} \cos (\alpha \pi / 2) z^{3 \alpha}+\left(b_{1}{ }^{2}-b_{2}{ }^{2}\right) z^{2 \alpha}-$ $2 b_{2} b_{3} \cos (\alpha \pi / 2) z^{\alpha}-b_{3}{ }^{2}$; one has

$$
h(\omega)=F(i \omega) \overline{F(i \omega)}-G(i \omega) \overline{G(i \omega)} .
$$


Differentiating both sides of (24) with respect to $\omega$, we obtain

$$
\begin{gathered}
h^{\prime}(\omega)=i\left[F^{\prime}(i \omega) \overline{F(i \omega)}-F(i \omega) \overline{F^{\prime}(i \omega)}\right. \\
\left.-G^{\prime}(i \omega) \overline{G(i \omega)}+G(i \omega) \overline{G^{\prime}(i \omega)}\right] .
\end{gathered}
$$

Differentiating both sides of (24) with respect to $\tau$ yields

$$
\begin{aligned}
& {\left[F^{\prime}(s)+G^{\prime}(s) e^{-s \tau}-\tau G(s) e^{-s \tau}\right] \frac{d s}{d \tau}-s G(s) e^{-s \tau}} \\
& \quad=0
\end{aligned}
$$

From (26), one obtains

$$
\begin{aligned}
\frac{d s}{d \tau} & =\frac{s G(s) e^{-s \tau}}{F^{\prime}(s)+G^{\prime}(s) e^{-s \tau}-\tau G(s) e^{-s \tau}} \\
& =\frac{s G(s)}{F^{\prime}(s) e^{s \tau}+G^{\prime}(s)-\tau G(s)} \\
& =\frac{s\left(\overline{F^{\prime}(s)} F(s)+\overline{G^{\prime}(s)} G(s)-\tau|G(s)|^{2}\right)}{\left|F^{\prime}(s) e^{s \tau}+G^{\prime}(s)-\tau G(s)\right|^{2}} .
\end{aligned}
$$

With $s=i \omega_{k}$, the above equality becomes

$$
\begin{aligned}
\left.\operatorname{Re}\left(\frac{d s}{d \tau}\right)\right|_{s=i \omega_{k}} & =\left.\frac{\operatorname{Re}\left(s\left(\overline{F^{\prime}(s)} F(s)+\overline{G^{\prime}(s)} G(s)-\tau|G(s)|^{2}\right)\right)}{\left|F^{\prime}(s) e^{s \tau}+G^{\prime}(s)-\tau G(s)\right|^{2}}\right|_{s=i \omega_{k}} \\
& =\frac{i \omega_{k}\left(\overline{F^{\prime}\left(i \omega_{k}\right)} F\left(i \omega_{k}\right)-F^{\prime}\left(i \omega_{k}\right) \overline{F\left(i \omega_{k}\right)}+\overline{G^{\prime}\left(i \omega_{k}\right)} G\left(i \omega_{k}\right)-G^{\prime}\left(i \omega_{k}\right) \overline{G\left(i \omega_{k}\right)}\right)}{2\left|F^{\prime}\left(i \omega_{k}\right) e^{i \omega_{k} \tau}+G^{\prime}\left(i \omega_{k}\right)-\tau G\left(i \omega_{k}\right)\right|^{2}} \\
& =\frac{\omega_{k} h^{\prime}\left(\omega_{k}\right)}{2\left|F^{\prime}\left(i \omega_{k}\right) e^{i \omega \tau}+G^{\prime}\left(i \omega_{k}\right)-\tau G\left(i \omega_{k}\right)\right|^{2}} .
\end{aligned}
$$

When $h^{\prime}\left(\omega_{k}\right)>0$, one gets $\left.\operatorname{Re}(d s / d \tau)\right|_{s=i \omega_{k}}>0$. Hence, the endemic equilibrium point $E_{1}$ of system (2) is locally asymptotically stable if $\tau<\tau_{0}$ and unstable if $\tau>\tau_{0}$. This completes the proof.

Remark 7. It is worth noting that there will be some future directions to apply our main results to more complex ones like models with time varying delay [36] and models with perturbed parameters [37] or to study the Hopf bifurcation of models with discrete and distributed delays [38].

\section{Numerical Simulations}

In this section, we give some numerical simulations for system (2) by using the method mentioned in $[39,40]$.

In Figure 1, we select parameters as $\alpha=0.98, k=5, \beta=$ $0.1, \mu=0.5, \gamma=2$, and $d=0.82$, with initial conditions $S_{0}=3.5, I_{0}=2$. After calculation, one obtains disease-free equilibrium point $E_{0}=(6.25,0)$ and $R_{0}=0.7622<1$. In (a), we take $\tau=0$, and in (b) we take $\tau=10$. According to Theorem 4, the disease-free equilibrium point of system (2) is locally asymptotically stable when $R_{0}<1$.

In Figure 2, the selected parameters are $\alpha=0.98, \tau=0$, $k=8, \beta=0.1, \mu=0.5, \gamma=4$, and $d=0.7$, with initial conditions $S_{0}=4, I_{0}=3$. After calculation, one obtains endemic equilibrium $E_{1}=(7,10)$ and $R_{0}=1.2857>1$. According to Theorem 5, if $\tau=0$ and $R_{0}>1$, the endemic equilibrium point of system (2) is locally asymptotically stable. The numerical simulation results are shown in Figure 2.

In Figures 3(a) and 3(b), we plotted the effect measure of immigration coefficient $\mu$ on susceptible and infected populations. The selected parameters are same as Figure 2 with initial conditions $S_{0}=6, I_{0}=3$. Values of $\mu$ are shown in the legend. From Figures 3(a) and 3(b), we observe that the number of susceptible individuals increases as $\mu$ increases at the beginning but is finally stable at the same fixed value. The number of infected individuals increases as $\mu$ increases. It shows that after the endemic formation, the number of the susceptible individuals increases as the number of floating population increases in the short term, but in the long run the number of susceptible individuals is the same, and only the number of infected individuals increases.

Figure 4 depicts the Hopf bifurcation of the endemic equilibrium. The parameters are taken as $k=10, \beta=0.1$, $r=10, \mu=0.6$, and $d=0.94$, with initial conditions $S_{0}=$ $12.4, I_{0}=9$. After calculation, one obtains $R_{0}=1.2857>1$, $h^{\prime}(\omega)>0$, and $E_{1}=(9.4,12)$. When $\alpha=0.96, \tau_{0}=12.6480$ is calculated. In (a), we let $\tau=12<\tau_{0}$, and in (b) $\tau=14>\tau_{0}$. (a) and (b) show Hopf bifurcation occurs at $\tau_{0}$. Then one selects different order $\alpha \in(0,1]$, we get different time delay, and the results are shown in (c). Figure 4(c) shows that as the value of $\alpha$ becomes smaller, the stability domain becomes larger. When $\alpha=0.9, \tau_{0}=15.1281$, and $\tau=14<\tau_{0}$, Figure $4(\mathrm{~d})$ shows that the endemic equilibrium point becomes stable.

\section{Conclusion}

In this paper, a fractional-order epidemic model with time delay is studied and stability and bifurcation of the model are analyzed. The results show that when $R_{0}<1$, the diseasefree equilibrium point is locally asymptotically stable for $\tau \geq 0$. And we get that when $R_{0}>1$ and $\tau=0$, the endemic equilibrium point is locally asymptotically stable. 


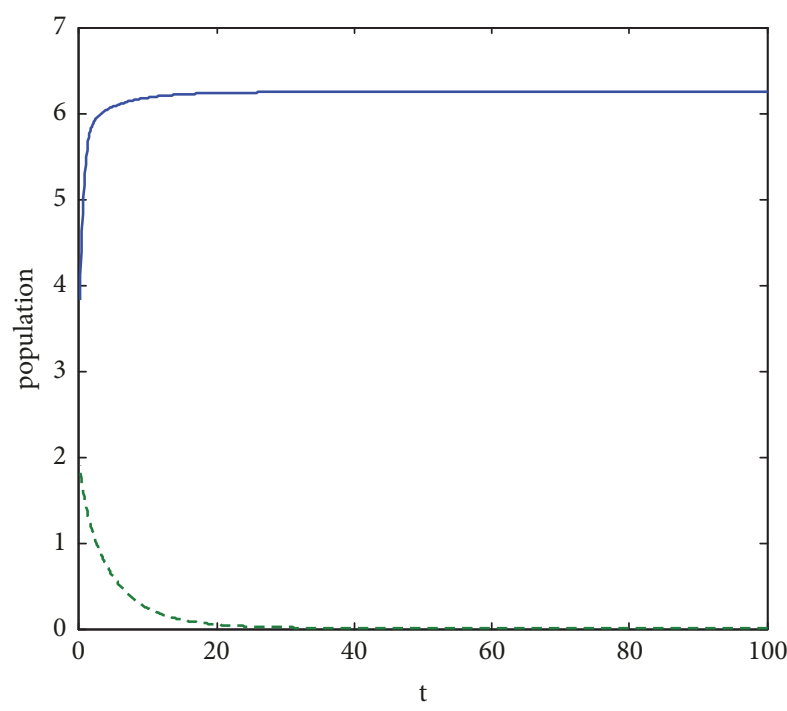

$-\mathrm{S}$

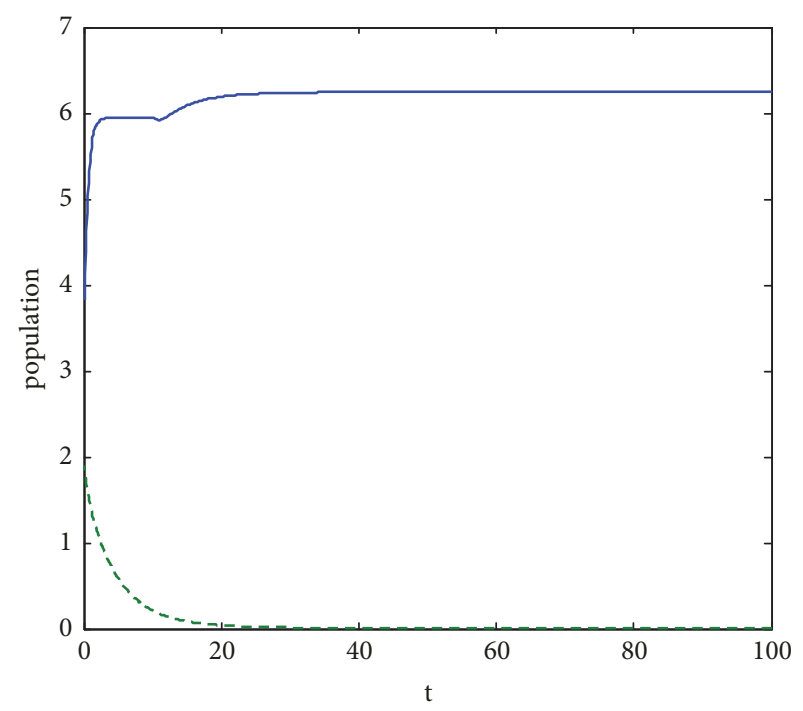

$-\mathrm{S}$

(a)

(b)

FIGURE 1: Stability of disease-free equilibrium point: (a) $\tau=0$; (b) $\tau=10$.

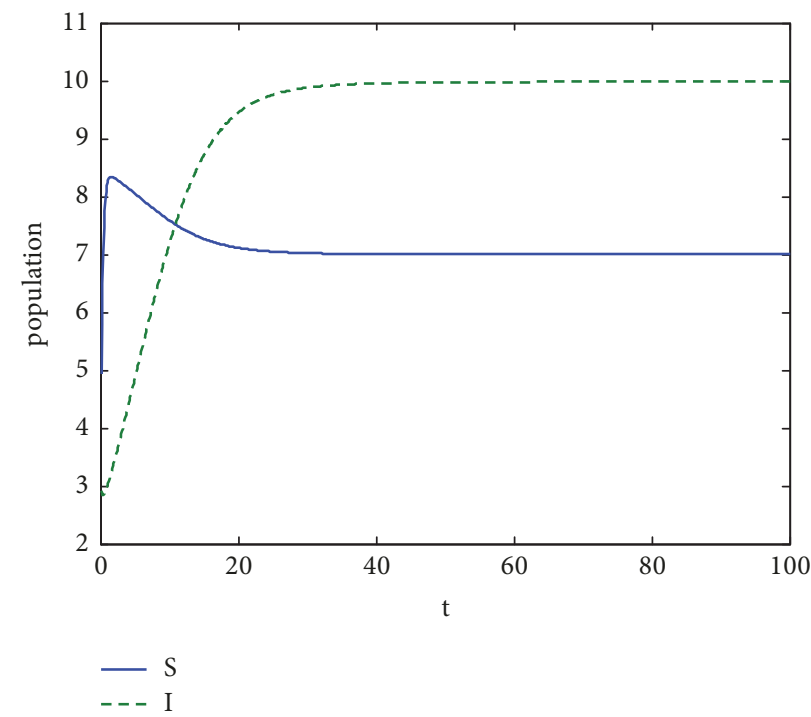

FIGURE 2: The stability of endemic equilibrium point when $\tau=0$.

According to Theorem 6, when $R_{0}>1$ and $h^{\prime}\left(\omega_{k}\right)>0$, the stability of the endemic equilibrium point changes at bifurcation point $\tau_{0}$. Some numerical simulations are given to verify the correctness of the theory, and stability region of model is related to the value of $R_{0}, \tau$, and fractional-order $\theta$.

\section{Data Availability}

No data were used to support this study.

\section{Conflicts of Interest}

The authors declare no conflicts of interest.

\section{Acknowledgments}

This work was supported by the National Natural Science Foundation of China (nos. 61573008, 61473178), the Natural Science Foundation of Shandong Province (no. 


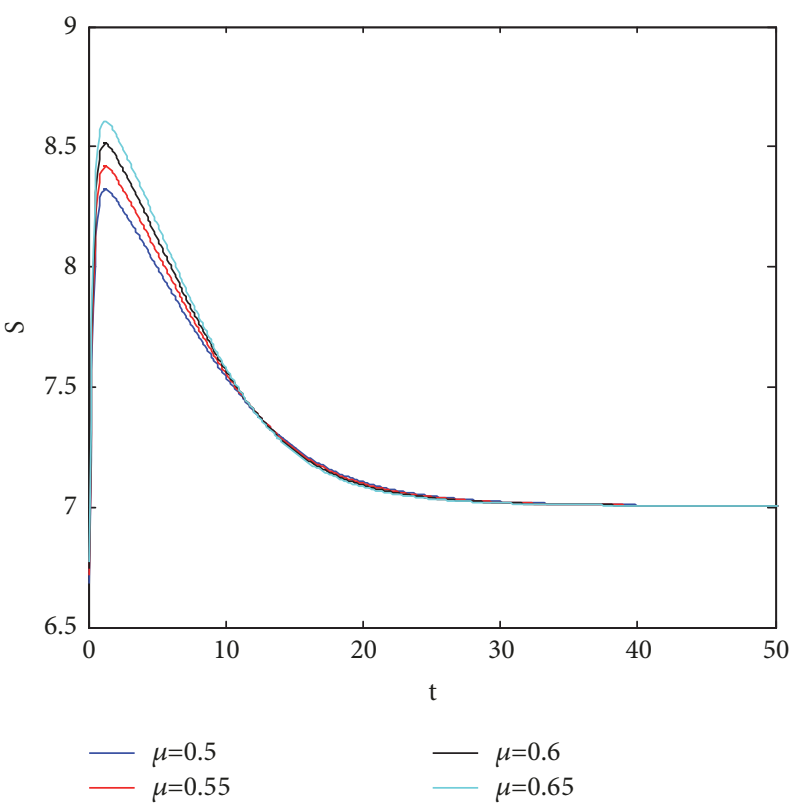

(a)

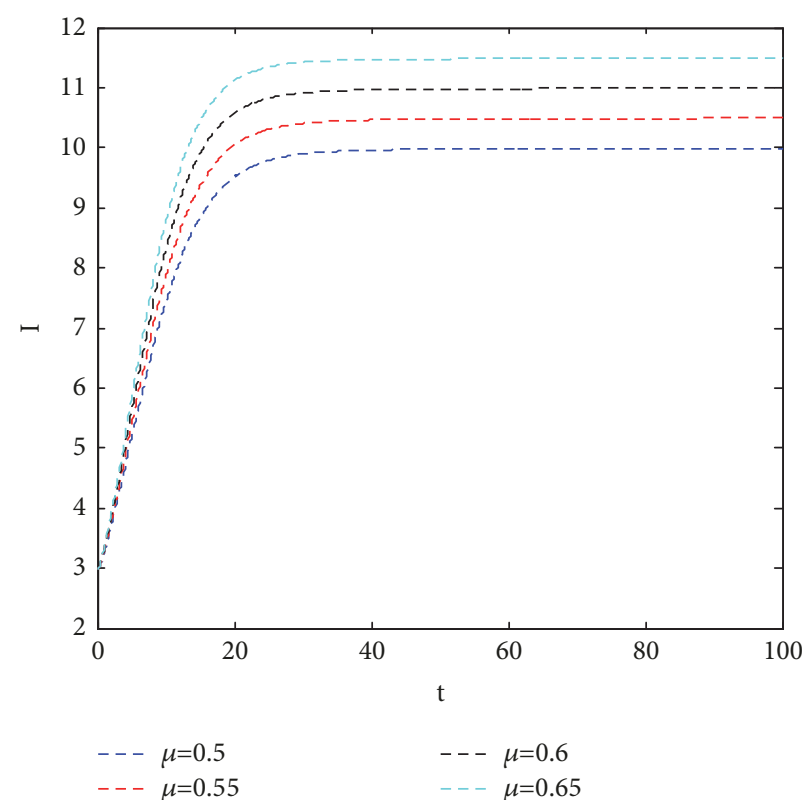

(b)

FIGURE 3: Effect of $\mu$ on $S$ on I, respectively, when $E_{1}$ is stable.
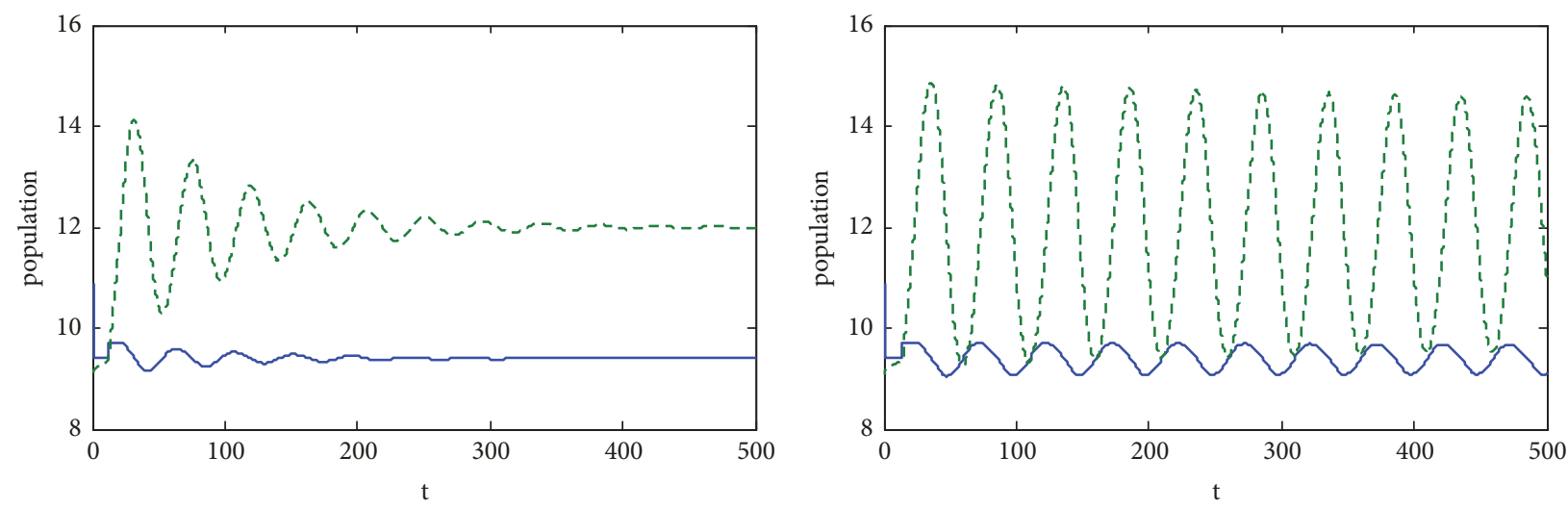

- S

(a)

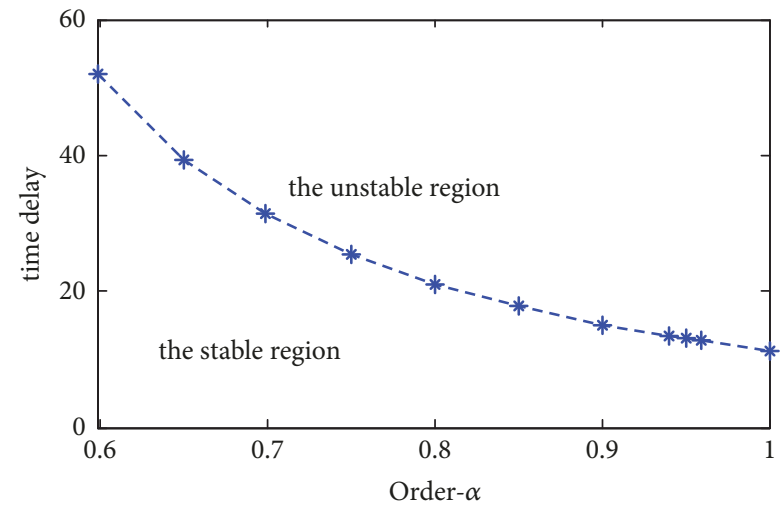

- S

(b)

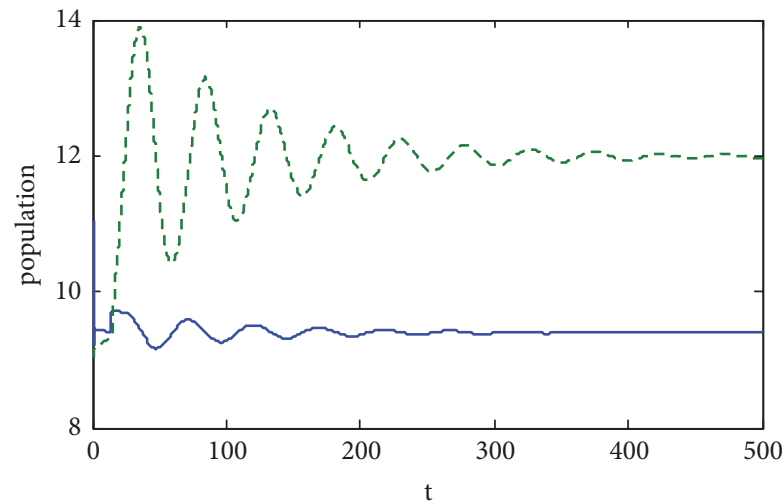

$-\mathrm{S}$

(c)

FIgURE 4: Hopf bifurcation of the endemic equilibrium point. 
ZR201709230160), Post-Doctoral Applied Research Projects of Qingdao (no. 2016115), and SDUST Research Fund (no. 2014TDJH102).

\section{References}

[1] W. O. Kermack and A. G. McKendrick, "A contribution to the mathematical theory of epidemics," Proceedings of the Royal Society A Mathematical, Physical and Engineering Sciences, vol. 115, no. 772, pp. 700-721, 1927.

[2] X. Meng and L. Zhang, "Evolutionary dynamics in a LotkaVolterra competition model with impulsive periodic disturbance," Mathematical Methods in the Applied Sciences, vol. 39, no. 2, pp. 177-188, 2016.

[3] X.-z. Meng, S.-n. Zhao, and W.-y. Zhang, "Adaptive dynamics analysis of a predator-prey model with selective disturbance," Applied Mathematics and Computation, vol. 266, pp. 946-958, 2015.

[4] J.-J. Cheng, Y. Liu, B. Shen, and W.-G. Yuan, "An epidemic model of rumor diffusion in online social networks," The European Physical Journal B, vol. 86, no. 1, Art. 29, 7 pages, 2013.

[5] Z. Bai and Y. Zhou, "Global dynamics of an SEIRS epidemic model with periodic vaccination and seasonal contact rate," Nonlinear Analysis: Real World Applications, vol. 13, no. 3, pp. 1060-1068, 2012.

[6] A. d'Onofrio, "Stability properties of pulse vaccination strategy in SEIR epidemic model," Mathematical Biosciences, vol. 179, no. 1, pp. 57-72, 2002.

[7] X. Zhou and J. Cui, "Analysis of stability and bifurcation for an SEIR epidemic model with saturated recovery rate," Communications in Nonlinear Science and Numerical Simulation, vol. 16, no. 11, pp. 4438-4450, 2011.

[8] D. Li, J. Cui, M. Liu, and S. Liu, "The evolutionary dynamics of stochastic epidemic model with nonlinear incidence rate," Bulletin of Mathematical Biology, vol. 77, no. 9, pp. 1705-1743, 2015.

[9] X.-Z. Li, G. Gupur, and G.-T. Zhu, "Threshold and stability results for an age-structured SEIR epidemic model," Computers \& Mathematics with Applications. An International Journal, vol. 42, no. 6-7, pp. 883-907, 2001.

[10] C. Sun and Y.-H. Hsieh, "Global analysis of an SEIR model with varying population size and vaccination," Applied Mathematical Modelling: Simulation and Computation for Engineering and Environmental Systems, vol. 34, no. 10, pp. 2685-2697, 2010.

[11] Z. Jiang, W. Ma, and J. Wei, "Global Hopf bifurcation and permanence of a delayed SEIRS epidemic model," Mathematics and Computers in Simulation, vol. 122, pp. 35-54, 2016.

[12] T. Zhang, X. Meng, and T. Zhang, "Global analysis for a delayed SIV model with direct and environmental transmissions," Journal of Applied Analysis and Computation, vol. 6, no. 2, pp. 479491, 2016.

[13] G. Ranjith Kumar, K. Lakshmi Narayan, and B. Ravindra Reddy, "Stability and Hopf bifurcation analysis of SIR epidemic model with time delay," ARPN Journal of Engineering and Applied Sciences, vol. 11, no. 3, pp. 1419-1423, 2016.

[14] I. Podlubny, Fractional Differential Equations : an Introduction to Fractional Derivatives, Fractional Differential Equations, to Methods of Their Solution and Some of Their Applications, Academic Press, San Diego, Calif, USA, 1999.
[15] Z. Wang, X. Wang, Y. Li, and X. Huang, "Stability and Hopf Bifurcation of Fractional-Order Complex-Valued Single Neuron Model with Time Delay," International Journal of Bifurcation and Chaos, vol. 27, no. 13, 1750209, 13 pages, 2017.

[16] L. Ferrara and D. Guegan, "Fractional seasonality: Models and Application to Economic Activity in the Euro Area," Aps March Meeting, vol. 74, no. 1, pp. 2400-2403, 2006.

[17] T. J. Freeborn, "A survey of fractional-order circuit models for biology and biomedicine," IEEE Journal on Emerging and Selected Topics in Circuits and Systems, vol. 3, no. 3, pp. 416-424, 2013.

[18] Y. Fan, X. Huang, Z. Wang, and Y. Li, "Nonlinear dynamics and chaos in a simplified memristor-based fractional-order neural network with discontinuous memductance function," Nonlinear Dynamics, vol. 93, no. 2, pp. 611-627, 2018.

[19] X. Huang, Y. Fan, J. Jia, Z. Wang, and Y. Li, "Quasi-synchronisation of fractional-order memristor-based neural networks with parameter mismatches," IET Control Theory \& Applications, vol. 11, no. 14, pp. 2317-2327, 2017.

[20] Z. Bai, Y. Chen, H. Lian, and S. Sun, "On the existence of blow up solutions for a class of fractional differential equations," Fractional Calculus and Applied Analysis, vol. 17, no. 4, pp. 1175-1187, 2014.

[21] Q. Song, X. Dong, Z. Bai, and B. Chen, "Existence for fractional Dirichlet boundary value problem under barrier strip conditions," Journal of Nonlinear Sciences and Applications. JNSA, vol. 10, no. 7, pp. 3592-3598, 2017.

[22] E. Ahmed and A. S. Elgazzar, "On fractional order differential equations model for nonlocal epidemics," Physica A: Statistical Mechanics and its Applications, vol. 379, no. 2, pp. 607-614, 2007.

[23] E. Ahmed, A. M. El-Sayed, and H. A. El-Saka, "Equilibrium points, stability and numerical solutions of fractional-order predator-prey and rabies models," Journal of Mathematical Analysis and Applications, vol. 325, no. 1, pp. 542-553, 2007.

[24] I. Ameen and P. Novati, "The solution of fractional order epidemic model by implicit Adams methods," Applied Mathematical Modelling: Simulation and Computation for Engineering and Environmental Systems, vol. 43, pp. 78-84, 2017.

[25] Y. Yan and C. Kou, "Stability analysis of a fractional differential model of HIV infection of $\mathrm{CD}^{+}{ }^{+}$T-cells with time delay," Mathematics and Computers in Simulation, vol. 82, no. 9, pp. 1572-1585, 2012.

[26] H. A. El-Saka, "Backward bifurcations in fractional-order vaccination models," Journal of the Egyptian Mathematical Society, vol. 23, no. 1, pp. 49-55, 2015.

[27] M. Javidi and N. Nyamoradi, "Dynamic analysis of a fractional order prey-predator interaction with harvesting," Applied Mathematical Modelling: Simulation and Computation for Engineering and Environmental Systems, vol. 37, no. 20-21, pp. 89468956, 2013.

[28] H. A. A. El-Saka, "The fractional-order SIS epidemic model with variable population size," Journal of the Egyptian Mathematical Society, vol. 22, no. 1, pp. 50-54, 2014.

[29] N. Supajaidee and S. Moonchai, "Stability analysis of a fractional-order two-species facultative mutualism model with harvesting," Advances in Difference Equations, Paper No. 372, 13 pages, 2017.

[30] D. Rostamy and E. Mottaghi, "Forward and Backward BIFurcation in a Fractional-Order SIR Epidemic Model with Vaccination," Iranian Journal of Science \& Technology, vol. 42, no. 2, pp. 663-671, 2018. 
[31] Z. Liu and P. Lu, "Stability analysis for HIV infection of CD4 $4^{+}$ T-cells by a fractional differential time-delay model with cure rate," Advances in Difference Equations, 2014:298, 20 pages, 2014.

[32] R. Chinnathambi and F. A. Rihan, "Stability of fractional-order prey-predator system with time-delay and Monod-Haldane functional response," Nonlinear Dynamics, vol. 92, no. 4, pp. 1637-1648, 2018.

[33] W. Deng, C. Li, and J. Lü, "Stability analysis of linear fractional differential system with multiple time delays," Nonlinear Dynamics, vol. 48, no. 4, pp. 409-416, 2007.

[34] I. Podlubny, Fractional Differential Equations, Academic Press, New York, NY, USA, 1999.

[35] C.-p. Li and Z.-g. Zhao, "Asymptotical stability analysis of linear fractional differential systems," Journal of Shanghai University (English Edition), vol. 13, no. 3, pp. 197-206, 2009.

[36] S. Jiao, H. Shen, Y. Wei, X. Huang, and Z. Wang, "Further results on dissipativity and stability analysis of Markov jump generalized neural networks with time-varying interval delays," Applied Mathematics and Computation, vol. 336, pp. 338-350, 2018.

[37] J. Wang, K. Liang, X. Huang, Z. Wang, and H. Shen, "Dissipative fault-tolerant control for nonlinear singular perturbed systems with Markov jumping parameters based on slow state feedback," Applied Mathematics and Computation, vol. 328, pp. 247-262, 2018.

[38] L. Li, Z. Wang, Y. Li, H. Shen, and J. Lu, "Hopf bifurcation analysis of a complex-valued neural network model with discrete and distributed delays," Applied Mathematics and Computation, vol. 330, pp. 152-169, 2018.

[39] Z. Wang, "A numerical method for delayed fractional-order differential equations," Journal of Applied Mathematics, vol. 2013, Article ID 256071, 7 pages, 2013.

[40] Z. Wang, X. Huang, and J. Zhou, "A numerical method for delayed fractional-order differential equations: based on G-L definition," Applied Mathematics \& Information Sciences, vol. 7, no. 2L, pp. 525-529, 2013. 


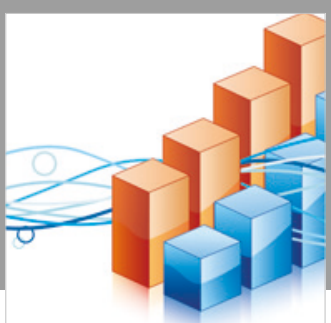

Advances in

Operations Research

\section{-n-m}
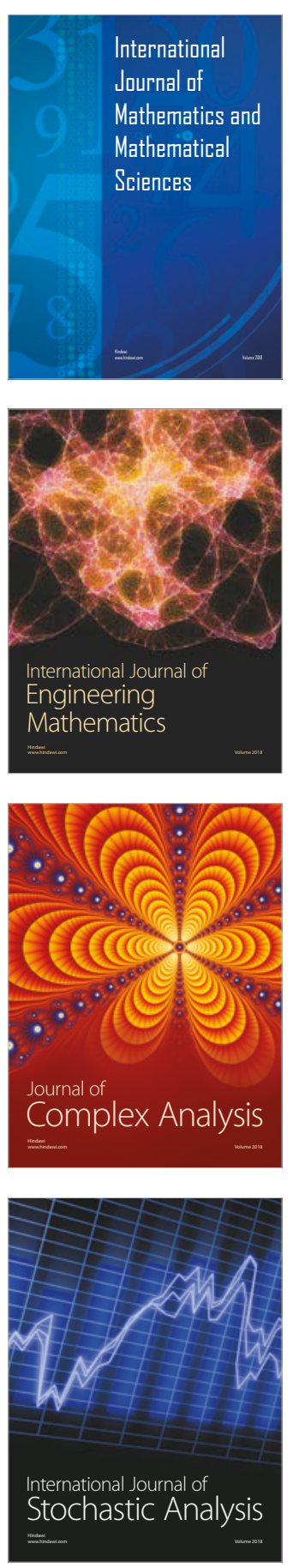
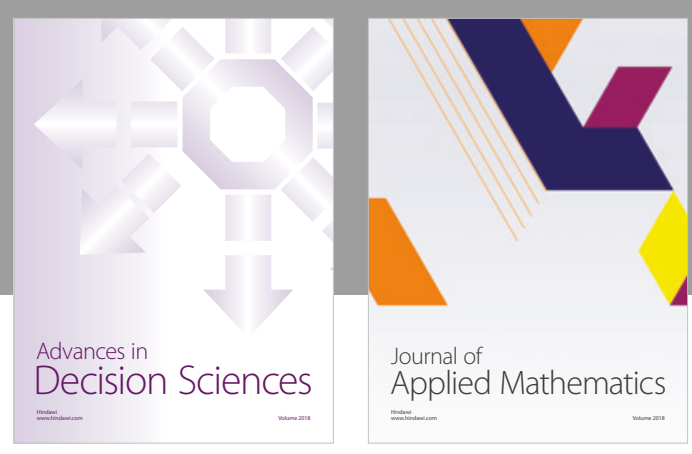

Journal of

Applied Mathematics
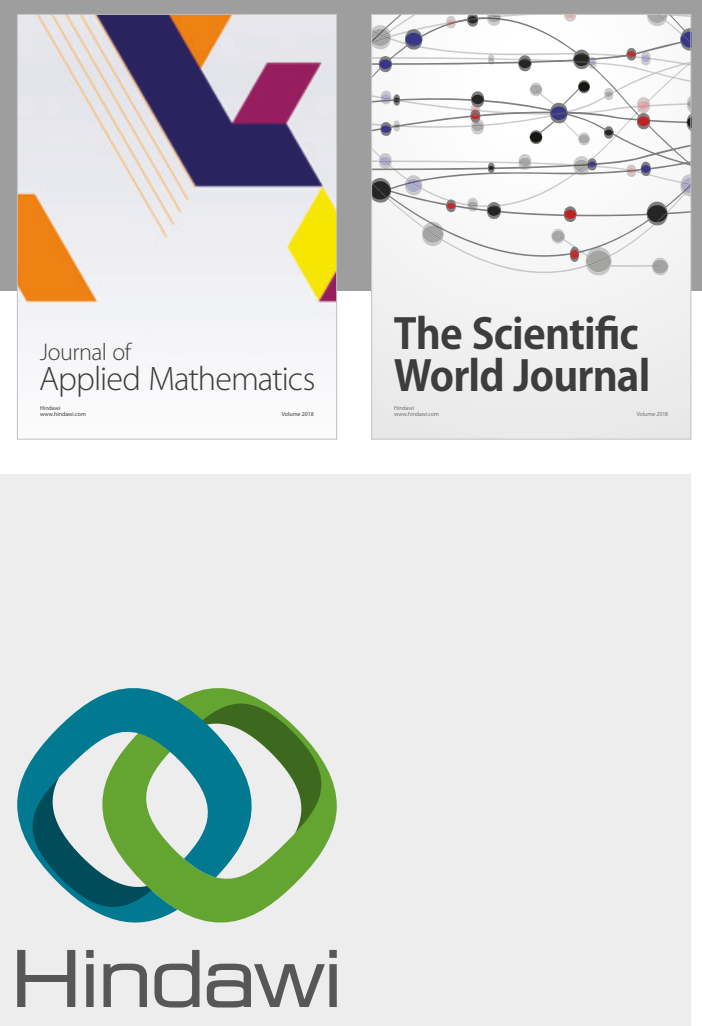

Submit your manuscripts at

www.hindawi.com

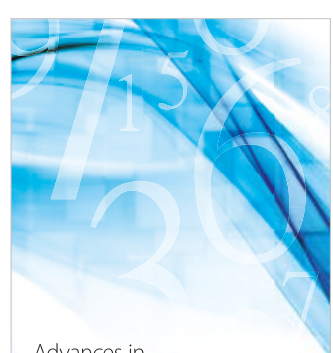

Advances in
Numerical Analysis
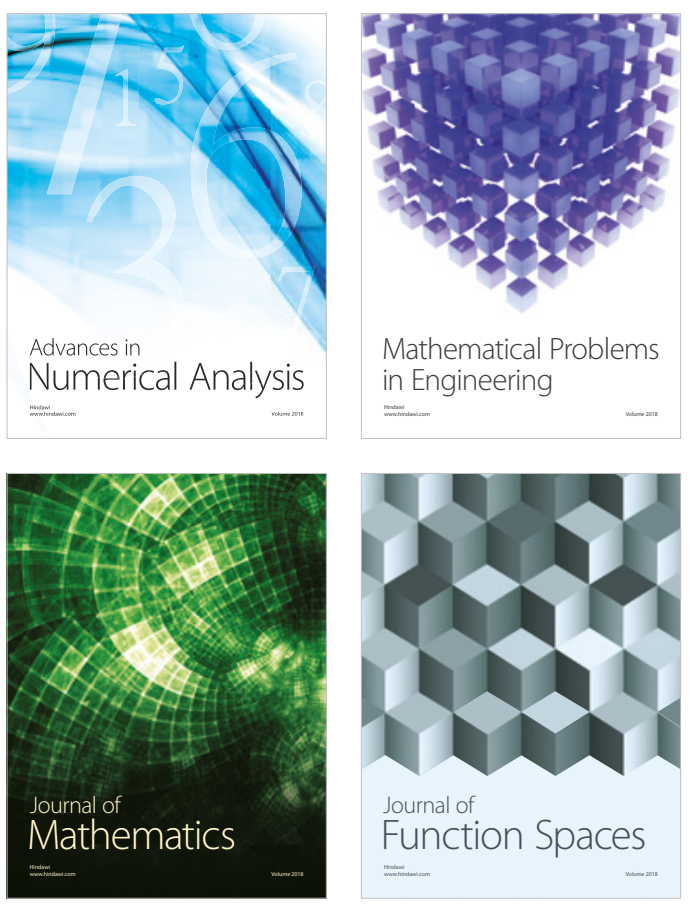

Mathematical Problems in Engineering

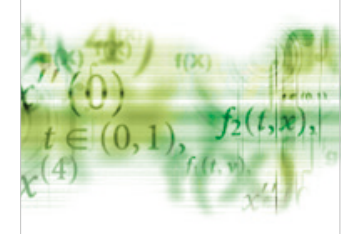

International Journal of

Differential Equations

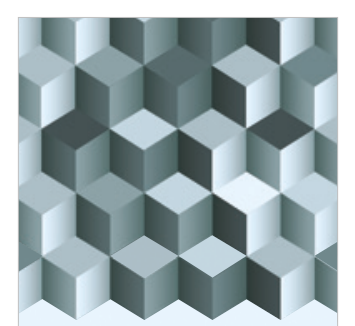

Journal of

Function Spaces

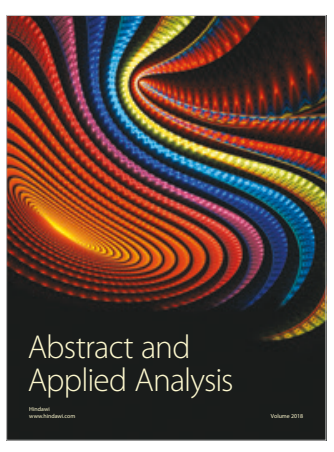

The Scientific

World Journal

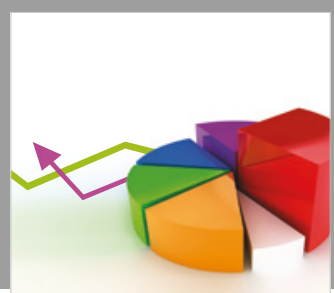

Journal of

Probability and Statistics
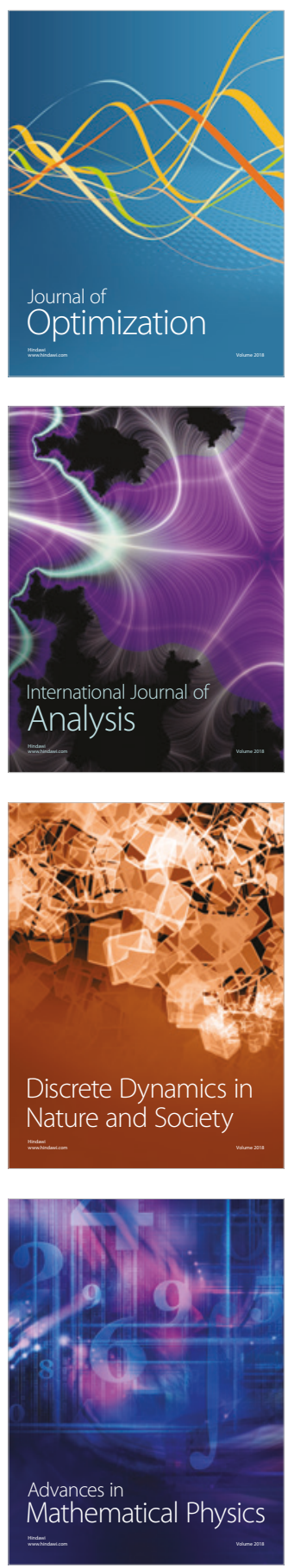